\title{
GROUPS SHARING SOME VARIETAL PROPERTIES WITH SUPERSOLUBLE GROUPS
}

\author{
ROLF BRANDL \\ (Received 25 November 1981; revised 21 March 1982) \\ Communicated by D. E. Taylor
}

\begin{abstract}
In this note a formation $\mathfrak{U}$ is considered which can be defined by a sequence of laws which 'almost' hold in every finite supersoluble group. The class $\mathfrak{u}$ contains all finite supersoluble groups and each group in $U$ has a Sylow tower.

It is shown that a finite group belongs to $\mathfrak{U}$ if and only if all of its subgroups with nilpotent commutator subgroup are supersoluble. A more general result concerning classes of this type finally proves that $\mathfrak{U}$ is a saturated formation.
\end{abstract}

1980 Mathematics subject classification (Amer. Math. Soc.): 20 D 10,20 F 16.

The classes of SA-groups (resp. SN-groups) which are by definition finite groups all of whose supersoluble subgroups are abelian (resp. nilpotent) have been the subject of several investigations. In [5] Napolitani has shown that $\mathrm{SN}$-groups are soluble. In [6] and [7] it has been shown by a detailed analysis of $\{p, q\}$ Hall subgroups that the classes of SA-groups and SN-groups are formations. Moreover (see [7, page 180]), the class of SN-groups is saturated and a Fitting class.

Our interest in classes of this type arose from an attempt to determine the structure of finite groups satisfying some law $\ddot{u}_{k}(x, y)=x^{(-1+y) \cdots(-k+y)}=1$ as used in [1, page $19 \mathrm{ff}]$. More precisely, we consider $\mathfrak{U}=\{G \mid G$ is finite and $\ddot{u}_{k}(G)=1$ for some positive integer $\left.k\right\}$. In [1, page $\left.19 \mathrm{ff}\right]$ it has been proved that $\mathfrak{U}$ contains all finite supersoluble groups and each group in $\mathfrak{U}$ has a Sylow tower. Moreover, $\mathfrak{U}$ contains groups of arbitrary Fitting length. We now have the following description of the class $\mathfrak{u}$.

Copyright Australian Mathematical Society 1983 
THEOREM 1. a) $\mathfrak{U}$ is a saturated formation, defined locally by formations $u(p)$ consisting of all soluble groups of exponent dividing $p-1$.

b) $\mathfrak{U}$ is the class of all finite groups all of whose subgroups with nilpotent commutator subgroup are supersoluble.

The proof of this result depends on Theorem 2. Before we state it, we introduce some notation.

Definition ([2, page 297]). Let $\mathfrak{A}$ and $\mathfrak{B}$ be classes of finite groups. Then $\mathfrak{A}[\mathfrak{B}$ is defined to be the class of all finite soluble groups $G$ satisfying:

$$
U \leqslant G \& U \in \mathfrak{A} \Rightarrow U \in \mathfrak{B} .
$$

Obviously, the classes of SA-groups and SN-groups are of this type.

THEOREM 2. Let $\mathfrak{A}$ and $\mathfrak{B}$ be formations of finite soluble groups, let $\mathfrak{A}$ be saturated. Then

a) If $\mathfrak{A}$ is $S$-closed then $\mathfrak{A}[\mathfrak{B}$ is an $S$-closed formation,

b) Let $\mathfrak{A}$ and $\mathfrak{B}$ be saturated and $S$-closed, and let $a(p)$ (resp. $b(p))$ be their full and integrated definitions. If $c(p)=a(p)[b(p)$ is a formation for all $p$, then $\mathfrak{C}=\mathfrak{A}[\mathfrak{B}$ is a saturated formation, locally defined by the $c(p)$.

c) If, moreover, all $c(p)$ are Fitting classes, then (5) is a Fitting class.

Most of the results in [6] and [7] are immediate consequences of Theorem 2, for example:

Corollary ([7, page $180 \mathrm{ff}])$. The class of $S N$-groups is a saturated Fitting formation. A local definition is given by the formations $t(p)$ of all soluble groups of order coprime to $p-1$.

Proof of Theorem 2. a) $Q$-closure of $\mathfrak{2}[\mathfrak{B}$ follows from [4, page 271] (see also [2, page 298]). $S$-closure and $D$-closure are obvious.

b) Let $\mathbb{C}^{+}$be the saturated formation, defined locally by the $c(p)$.

We first prove $\mathbb{S}^{+} \subseteq \mathfrak{A}\left[\mathfrak{B}\right.$. Let $G \in \mathbb{C}^{+}$and let $U \leqslant G$ with $U \in \mathfrak{A}$. Now the $c(p)$ are $S$-closed and so [3, page $351 \mathrm{f}]$ yields $U \in C^{*}$. Let $p$ be a prime. Then $U / O_{p^{\prime}, p}(U) \in a(p)$. As $U \in \mathfrak{C}^{+}$, we have $U / O_{p^{\prime}, p}(U) \in a(p)[b(p)$. Hence $U / O_{p^{\prime}, p}(U) \in b(p)$ and we arrive at $U \in \mathfrak{B}$. This implies $G \in \mathfrak{U}[\mathfrak{B}$.

Conversely, let $G \in \mathfrak{A}[\mathfrak{B}$ and let $H / K$ be a $p$-chief factor of $G$. We need to show $G / C_{G}(H / K) \in c(p)$.

Let bars denote factors modulo $K$. Let $A=\bar{G} / C(\bar{H}) \cong G / C_{G}(H / K)$. Furthermore, let $U \leqslant A$ with $U \in a(p)$. We consider the splitting extension $S=\bar{H} A$ and 
its subgroup $V=\bar{H} U$. By [4, page 707], we have $S \in \mathfrak{A}[\mathfrak{B}$ and so

$$
V \in \mathfrak{A}[\mathfrak{B} .
$$

Now, by definition, $U$ acts faithfully on $\bar{H}$. So we have $V / O_{p^{\prime}, p}(V)=V / O_{p}(V)$ $\cong U / O_{p}(U) \in a(p)$ and for all primes $q \neq p: V / O_{q^{\prime}, q}(V) \cong U / O_{q^{\prime}, q}(U) \in a(q)$ as $U \in a(p) \subseteq \mathfrak{A}$. This implies $V \in \mathfrak{A}$ and by (*), we have $V \in \mathfrak{B}$. This implies $U / O_{p}(U) \cong V / O_{p^{\prime}, p}(V) \in b(p)$. As the $b(p)$ form a full local definition of $\mathfrak{B}$, we conclude that $U \in b(p)$ as required. Done.

c) This follows from $b$ ) and [3, page $350 \mathrm{f}]$.

EXAMPLE. Let $\mathfrak{A}$ be the class of finite soluble groups all of whose nilpotent subgroups are abelian ( $\mathfrak{A}$ is the class of $A$-groups, see [4, page $751 \mathrm{ff}]$ ) and let $\mathfrak{B}$ be the saturated formation of supersoluble groups. Then the nonnilpotent extension of a quaternion group by a cycle of order three is in $\mathfrak{A}[\mathfrak{B}$, but its Frattini factor is not. So, $\mathfrak{A}[\mathfrak{B}$ need not be $Q$-closed in general, even if $\mathfrak{B}$ is saturated. So, in Theorem 2, some extra assumptions on $\mathfrak{A}$ resp. $a(p)[b(p)$ are necessary.

Proof of Theorem 1. b) We first show that finite groups all of whose subgroups with nilpotent derived group are supersoluble possess a Sylow tower. Let $G$ be a minimal counterexample to this assertion and let $p$ be the least prime dividing the order of $G$. Then, by [4, page 434, Satz 5.4], $G$ is a splitting extension of a $p$-group by a cyclic $q$-group where $q>p$. As $G^{\prime}$ is nilpotent, $G$ is supersoluble by assumption. So $G$ is $q$-closed, hence $G$ is nilpotent which contradicts the choice of $G$.

We now prove that $G$ satisfies almost all laws $\ddot{u}_{k}{ }^{-}=1$. Let $N$ be a minimal normal subgroup of $G$. By the above, $G$ is soluble and so $N$ is abelian. By induction on the order of $G$ we may assume that $\ddot{u}_{k}(G) \leqslant N$ for almost all $k$. It suffices to show that for each $n \in N$ and each $g \in G$ there exists $j \geqslant k$ with $n^{(-k+g) \cdots(-j+g)}=1$. As the derived group of $U=\langle N, g\rangle$ is nilpotent, the assumption on $G$ yields that $U$ is supersoluble. Finally the proof of [1, Satz 5.2] yields the desired result. So $G$ satisfies almost all laws $\ddot{u}_{k}=1$.

The reverse implication follows from [1, Hauptsatz 5.5].

a) By b), we have $\mathfrak{U}=\mathscr{R}[\ddot{\mathfrak{U}}$, where $\mathfrak{\Re}$ denotes the class of finite groups with nilpotent commutator subgroup and $\ddot{\mathfrak{U}}$ is the class of finite supersoluble groups. A full and integrated local definition for $\bar{R}$ is provided by formations $a(p)=\{X \mid$ $X / O_{p}(X)$ is abelian $\}$. Similarly, $\ddot{\mathfrak{u}}$ is defined by $b(p)=\left\{X \mid X / O_{p}(X)\right.$ is abelian of exponent $p-1\}$. So $a(p)\left[b(p)=\left\{X \mid X / O_{p}(X)\right.\right.$ is soluble of exponent $\left.p-1\right\}$ and our assertion follows from Theorem $2 b$ ). 


\section{References}

[1] R. Brandl, 'Zur Theorie der untergruppenabgeschlossenen Formationen: Endliche Varietäten.' J. Algebra 73 (1981), 1-22.

[2] R. Carter, B. Fischer and T. Hawkes, 'Extreme classes of finite groups,' J. Algebra 9 (1968), 285-313.

[3] K. Doerk, 'Zur Theorie der Formationen endlicher auflösbarer Gruppen,' J. Algebra 13 (1969), 345-373.

[4] B. Huppert, Endliche Gruppen I, Springer-Verlag, Berlin, Heidelberg, New York, (1967).

[5] F. Napolitani, 'Sui gruppi finiti privi di sottogruppi supersolubili non speciali,' Rend. Sem. Mat. Univ. Padova 39 (1967), 291-295.

[6] A. Scarselli, 'Sui gruppi a sottogruppi supersolubili abeliani,' Atti Accad. Naz. Lincei Rend. Cl. Sci. Fis. Mat. Natur. 60 (1976), 564-569.

[7] A. Scarselli, 'SA-gruppi e SN-gruppi,' Boll. Un. Mat. Ital. A (5) 14 (1977), 174-182.

\section{Mathematisches Institut}

Am Hubland 12

D-8700 Würzburg

Germany 